\title{
Associative transfer in verbal paired-associate learning as a function of stimulus similarity'
}

ANHE C. SARAVQ, FELS RESEARCH INSTITUTE LOUIS E. PRICE, UNIVERSITY OF NEW MEXICO

Associative transfer was explored in the $A-B, A-C$ and $A-B, A-B r$ paradigms under three levels of stimulus similarity using an A-B, C-D control comparison. Three groups, differing in degree of structural similarity, learned two mixed lists of CVC trigrams by the anticipation method. A total of 90 sixth grade $S$ s were used. The effect of paradigms was highly significant, with both the A-B, A-C and A-B, A-Br paradigms demonstrating negative transfer. Some evidence was found for increasing negative transfer as stimulus similarity increased.

The purpose of the present investigation was to assess the effects of stimulus simllarity on transfer in the $A-B, A-C$ and $A-B, A-B r$ paradigms, both of which generally result in associative inteference when evaluated against an A-B, C-D control for nonspecific transfer (Besch \& Reynolds, 1958), A secondary purpose was to develop a technique for using nonsense syllables in a paired-associate learning task with children.

Stimulus similarity, defined in terms of common elements in CVC trigrams, has been shown to retard learning within a single list (Goss \& Nodine, 1965, Ch. 5; Goss, Nodine, Gregory, Taub, \& Kennedy, 1962). Kazura (1961), using an A-B, A-C paradigm, found a condition of high similarity to result in lowered performance when compared with that of low similarity, however, since a control for nonspecific transfer was not included in the design, the amount of interference due to increased similarity cannot be assessed. The present study, then, represents an attempt to determine the relative effects of stimulus similarity on transfer in both the A-C and A-Br paradigms with nonspecific transfer controlled. Method

Ninety Ss, ranging in approximate age from 10 to 13 years, were selected from the sixth grade of a school in Chicopee, Massachusetts. A Hunter Card Master was used to present the stimuli which were displayed on white plastic cards for this purpose. The stimuli and responses were nonword CVCs with Archer (1960) association values from 84 to 94 . Stimulus members of high similarity shared two letters in common (e.g., NAL, JAL), those of medium similarity had one letter in common (e.g., DEK, MES) and those of low similarity shared no elements (e.g., WIP, ROG). The response members were of low similarity with one or no letters in common. Similarity between the stimulus and response members of a pair was minimal.

Within each level of similarity, manipulated between
Ss, two six pair lists defined the three paradigms, with two pairs each representing the $\mathrm{A}-\mathrm{C}, \mathrm{A}-\mathrm{Br}$, and C-D conditions. Three counterbalancing forms were constructed for each level of similarity to control for possible differential difficulty of the stimulus response pairs representing the three paradigms.

The $S$ was seated in front of the apparatus and told to anticipate each response syllable by pronouncing it prior to its exposure. For the first trial $E$ pronounced all response syllables. Preliminary work had shown that sixth grade children learn these syllables faster by pronouncing rather than spelling them, possibly due to better response integration in the former case. Ss were run to a criterion of five correct of six responses plus an additional trial, or for a maximum of 25 trials. List 1 cards were then removed and List 2 cards were inserted in the apparatus. During this interval of less than $30 \mathrm{sec}$. Ss were told that the procedure for the new list would be the same as before. All Ss were given 12 trials on the second list.

The anticipation interval, joint presentation interval, and interstimulus interval were 4,2 , and $2 \mathrm{sec}$, respectively, for List 1 and 2, 2, and $2 \mathrm{sec}$ for List 2. Intertrial intervals of $8 \mathrm{sec}$ in List 1 and $6 \mathrm{sec}$ in List 2 were achieved by inserting a blank card at the end of each trial.

\section{Resulis and Discussion}

List 1. The mean number of trials to criterion for low, medium, and high conditions of similarity were $13.6,15.0$, and 15.9 , respectively. Separate analyses of variance were performed on the trials-to-criterion measure and the post-criterion-trial measure, with similarity and counterbalancing forms as between-Ss variables. The differences among the means for low, medium, and high similarity were not significant, nor was the main effect for counterbalancing forms or the interaction of forms with similarity. $A \mathrm{X}^{2}$ analysis of the number of Ss reaching criterion showed no significant differences among the nine subgroups.

List 2. Table 1 presents the means and standard deviations of the correct responses for the A-C, A-Br, and $C-D$ paradigms for the three counterbalancing forms within each level. An analysis of variance with similarity and counterbalancing forms as between-Ss variables and paradigms and trials as within-Ss comparisons was performed on the correct responses given in List 2. This analysis showed paradigms to be highly significant ( $F=24.50, d f=2 / 162, p<.001)$. Over all levels of similarity, consistently higher performance occurred with the $C-D$ paradigm than with 
Table 1.

Means and SDs for Correct Responses in the Transfer Task

\begin{tabular}{|c|c|c|c|c|c|c|c|c|c|}
\hline \multirow{3}{*}{ Paradigm } & \multirow{3}{*}{ Form } & \multicolumn{6}{|c|}{ Similarity } & \multirow[t]{3}{*}{ Mean } & \multirow[t]{3}{*}{ SD } \\
\hline & & \multicolumn{2}{|c|}{ High } & \multicolumn{2}{|c|}{ Medium } & \multicolumn{2}{|c|}{ Low } & & \\
\hline & & Mean & SD & Mean & SD & Mean & SD & & \\
\hline \multirow{4}{*}{$A-B, A-B r$} & 1 & 5.9 & 4.53 & 8.1 & 4.38 & 9.1 & 7.03 & \multirow{4}{*}{7.3} & \multirow{4}{*}{4.65} \\
\hline & 2 & 8.3 & 2.31 & 7.0 & 4.92 & 6.1 & 5.40 & & \\
\hline & 3 & 7.0 & 3.52 & 7.7 & 3.68 & 11.8 & 3.36 & & \\
\hline & $M$ & 7.1 & 3.59 & 7.6 & 4.23 & 9.0 & 5.78 & & \\
\hline \multirow{4}{*}{$A-B, A \cdot C$} & 1 & 6.5 & 3.52 & 8.7 & 4.21 & 8.2 & 3.45 & \multirow{4}{*}{7.5} & \multirow{4}{*}{4.11} \\
\hline & 2 & 7.1 & 2.96 & 8.6 & 4.71 & 8.9 & 5.04 & & \\
\hline & 3 & 6.8 & 5.07 & 6.4 & 3.97 & 6.6 & 4.14 & & \\
\hline & $M$ & 6.8 & 3.87 & 7.9 & 4.30 & 7.9 & 4.22 & & \\
\hline \multirow{5}{*}{$A \cdot B, C-D$} & 1 & 13.8 & 2.85 & 12.1 & 4.65 & 12.2 & 4.98 & \multirow{5}{*}{10.8} & \multirow{5}{*}{5.21} \\
\hline & 2 & 5.8 & 3.91 & 8.8 & 5.05 & 7.2 & 4.21 & & \\
\hline & 3 & 13.6 & 4.90 & 11.3 & 4.85 & 13.2 & 5.53 & & \\
\hline & $M$ & 11.0 & 5.41 & 10.7 & 4.89 & 10.9 & 5.46 & & \\
\hline & TOT & 24.9 & 8.30 & 26.2 & 9.67 & 27.71 & 12.44 & & \\
\hline
\end{tabular}

the other paradigms, indicating negative transfer for both the $\mathrm{A}-\mathrm{C}$ and $\mathrm{A}-\mathrm{Br}$ paradigms. Although more correct responses were given under the $A-C$ than under the A-Br paradigm, a t-test of these means did not reach significance $(p>.10)$. Negative transfer for the $\mathrm{A}-\mathrm{C}$ and $\mathrm{A}-\mathrm{Br}$ paradigms increased with increasing stimulus similarity, particularly from medium to high similarity for counterbalancing Forms 1 and 3; counterbalancing Form 2 showed positive transfer with increasing stimulus similarity for the $\mathrm{A}-\mathrm{Br}$ paradigm. This effect, the Form by Paradigms by Similarity interaction, was significant $(F=2.02$, $\mathrm{df}=$ $8 / 162, p<.05)$. Performance over trials showed a significant increase in the transfer task $(F=230.60$, $\mathrm{df}=5 / 405, \mathrm{p}<.001)$, and a significant interaction with paradigms $(F=3.64, \mathrm{df}=10 / 810, \mathrm{p}<.01)$.

Although a consistent trend of decreasing performance with increases in stimulus similarity was observed in both the acquisition and transfer lists, in neither case was the main effect of this variable significant. However, the common letters defining any level of similarity always appeared in the same position in each of the syllables and it is possible that the effects of similarity were attenuated through reliance solely on the letters which differed among stimuli.

Since the means for the transfer task showed a difference due to similarity, it seemed likely that an additional uncontrolled factor was inflating the error term in the transfer task. An analysis of the responses common to both lists showed that two responses which appeared together in all forms and paradigms of the transfer task resulted in significantly poorer performance than the others. The greater difficulty of these items should not have affected the overall significance of paradigms since this difference would be washed out by the counterbalancing if the effect were additive and confined to the second task. Within any paradigm, however, differential performance might be expected on the different forms. In the $\mathrm{A}-\mathrm{Br}$ paradigm less negative transfer occurred with the two difficult responses than with the other two counterbalancing forms. In fact, these responses showed positive transfer under the high similarity condition. Since only the $\mathrm{A}-\mathrm{Br}$ paradigm provides practice of the transfer responses in the first list, it may be that familiarization on these two responses reduced the negative transfer effect. Thus the significant Form by Similarity by Paradigms interaction suggests that the more difficult the task, the greater the effect of response familiarization. An analysis of covariance, using the ranks of acquisition-task performance on the common responses as the covariate, decreased the within-Ss error term slightly, leaving the levels of significance of the effects unchanged, again suggesting a nonadditive effect of the covariate, response difficulty.

It is not known what made the two trigrams so much harder to learn than the others, since these responses were within one point of the others in the Archer association-value list. This points up the difficulties in generalizing adult norms to studies of learning in children. The need for a list of nonsense syllables standardized on children for association values and/or difficulty in learning is particularly acute since the learning abilities of children necessitate relatively short lists, thus reducing the possible sample of items used.

Within the above limitations, the significant negative transfer effect for children, using CVC trigrams as stimuli, hitherto unreported, confirms the results of other studies which have used adults as Ss, adjectives as stimuli, or both.

\section{References}

Archer, E. J. Rewevaluation of the meaningfulness of all possible CVC trigrams. Psychol. Monogr., 1960, 74, (Whole No. 497).

Besch, N. F., \& Reynolds, W. F. Associative interference in verbal paired-associate learning. J. exp. Psychol., 1958, 55, 554-558.

Goss, A. E., \& Nodine, C. F. Paired-associates learning: The role of meaningfulness, similarity, and familiarization. New York: Academic Press, 1965.

Goss, A. E., Nodine, C. F., Gregory, B. N., Taub, H. A., \& Kennedy, K. E. Stimulus characteristics and percentage of occurrence of response members in paired-associate learning. Psychol, Monogr., 1962, 76 (Whole No. 531).

Kazura, $\mathbf{k}$. The mechanism of generalization and differentiation in paired-associate learning. Jap. J. Psychol., 1961, 32, 287-294. Notes

1. This paper is based on a Master's problem by the first author and was presented at the annual meeting of the Eastern Psychological Association, Philadelphia, in April, 1964. 